\title{
FUNDAMENTAL SYSTEMS AND SOLUTIONS OF NONHOMOGENEOUS EQUATIONS FOR A PAIR OF MIXED LINEAR ORDINARY DIFFERENTIAL EQUATIONS
}

\author{
M. VENKATESULU and T. GNANA BHASKAR
}

(Received 17 January 1989)

Communicated by A. J. Pryde

\begin{abstract}
Two different ordinary differential operators $L_{1}$ and $L_{2}$ (not of the same order) defined on two adjacent intervals $I_{1}$ and $I_{2}$, respectively, with certain mixed conditions at the interface are considered. These problems are encountered in the study of 'acoustic waveguides in ocean', 'transverse vibrations in nonhomogeneous strings', etc. A complete set of physical conditions on the system give rise to three types of (selfadjoint) boundary value problems associated with the pair $\left(L_{1}, L_{2}\right)$. In a series of papers, a systematic study of these new classes of problems is being developed. In the present paper, we construct the fundamental systems and exhibit the forms of solutions of nonhomogeneous problems associated with the pair $\left(L_{1}, L_{2}\right)$.
\end{abstract}

1980 Mathematics subject classification (Amer. Math. Soc.) (1985 Revision): 34 A 99.

\section{Introduction}

In the studies of 'acoustic waveguides in ocean', 'optical fiber transmission', 'transverse vibrations of nonhomogeneous strings', 'one dimensional heat conduction in composite rods', etc., we encounter a new class of problems of the type

$$
L_{1} x=\frac{1}{r_{1}} \sum_{k=0}^{n} P_{k} \frac{d^{k} x}{d t^{k}}=\lambda x
$$

(C) 1990 Australian Mathematical Society $0263-6115 / 90 \$ A 2.00+0.00$ 
defined on an interval $I_{1}$ and

$$
L_{2} y=\frac{1}{r_{2}} \sum_{k=0}^{m} Q_{k} \frac{d^{k} y}{d t^{k}}=\lambda y
$$

defined on the adjacent interval $I_{2}$ where $I_{1}$ and $I_{2}$ have a common boundary point $t=b, \lambda$ is an unknown constant (eigenvalue), and the functions $x, y$ are required to satisfy certain mixed conditions at the interface $t=b$. In most of the cases, the complete set of physical conditions on the system give rise to selfadjoint boundary value problems associated with the pair $\left(L_{1}, L_{2}\right)$. We may broadly classify these boundary value problems into three types, namely (i) where the values of $x$ and $y$ at the interface point $t=b$ are not explicitly related to each other, (ii) where $x$ and $y$ satisfy continuity conditions at $t=b$, and (iii) where $x$ and $y$ satisfy certain matching conditions at $t=b$. In the literature, to the authors' knowledge, there does not exist a systematic mathematical study of these problems. In the present work, we shall construct fundamental systems, and shall establish the forms of solutions of nonhomogeneous equations associated with $\left(L_{1}, L_{2}\right)$ for each one of the three types of problems mentioned above.

In our earlier papers, we have studied the solutions of initial value problems [6], and characterized all selfadjoint boundary value problems [7] associated with the pair $\left(L_{1}, L_{2}\right)$. The next fundamental aspect of this new class of problems is the study of Green's functions and generalized Green's functions associated with $\left(L_{1}, L_{2}\right)$ which in turn helps the study of resolvents of selfajdoint boundary value problems associated with $\left(L_{1}, L_{2}\right)$. But before embarking upon these studies, it is essential to know about the fundamental systems, and the form of solutions of nonhomogeneous equations for the pair $\left(L_{1}, L_{2}\right)$, which we shall consider here. To know the physical situations where these new classes of problems arise, we may refer to $[1,3$, $4,5]$.

Before indicating the sectionwise splitup of the work we shall introduce a few notations and make some assumptions. For an interval $I$ of $R$ and for any positive integer $k$, let $A C^{k}(I)$ denote the space of all complex valued functions $f$ which have $(k-1)$ derivatives in $I$ and the $(k-1)$ th derivative is absolutely continuous over each compact subinterval of $I$. For a function $f$, let $f^{(k)}$ denote the $k$ th derivative of $f$, if it exists. Let $I_{1}=[a, b]$ or $(a, b]$ and $I_{2}=[b, c]$ or $[b, c),-\infty \leq a<b<c \leq+\infty$. For a function $f \in A C^{n}\left(I_{1}\right)$, let $\tilde{f}(t)=\operatorname{column}\left(f(t), \ldots, f^{(n-1)}(t)\right), t \in I_{1}$, and for a function $f \in A C^{m}\left(I_{2}\right)$, let $\tilde{\tilde{f}}(t)=\operatorname{column}\left(f(t), \ldots, f^{(m-1)}(t)\right), t \in I_{2}$. Let $I=I_{1} \cup I_{2}$. For a function $f$ defined on $I$, let $f / I_{i}$ denote the restriction of $f$ to $I_{i}, i=1,2$. Let $\mathbb{C}^{k}$ denote the $k$-dimensional complex space 
whose elements we take to be column vectors. If $\alpha \in \mathbb{C}^{k_{1}}$ and $\beta \in \mathbb{C}^{k_{2}}$, then the column $(\alpha, \beta)$ denotes the $\left(k_{1}+k_{2}\right)$ column vector formed by the components of $\alpha$ followed by the components of $\beta$ taken in that order.

Assumption 1. Let $L_{1}=\frac{1}{r_{1}} \sum_{k=0}^{n} P_{k} d^{k} / d t^{k}$ and $L_{2}=\frac{1}{r_{2}} \sum_{k=0}^{m} Q_{k} d^{k} / d t^{k}$, where $r_{1}, P_{k}, k=0,1, \ldots, n$, are continuous complex valued functions defined on $I_{1}$ with $r_{1}(t)>0$ and $P_{n}(t) \neq 0$ for all $t \in I_{1}$, and $r_{2}, Q_{k}$, $k=0,1, \ldots, m$, are continuous complex valued functions defined on $I_{2}$ with $r_{2}(t)>0$ and $Q_{m}(t) \neq 0$ for all $t \in I_{2}$. For the sake of definiteness, we assume that $n \geq m$.

Assumption 2. Let $g$ be a Lebesgue measurable complex valued function defined on $I$ which is integrable over every compact subinterval of $I$. Let $g_{i}=g / I_{i}, i=1,2$.

Assumption 3. Let $A$ and $B$ be $(m \times n)$ and $(m \times m)$ matrices with complex entries, respectively, such that the range of $A$ equals the range of $B$, and (hence) $\operatorname{rank} A=\operatorname{rank} B=d(\leq m)$.

Consider the homogeneous equation

$$
\left(L_{1}, L_{2}\right) f=0
$$

and the nonhomogeneous equation

$$
\left(L_{1}, L_{2}\right) f=g .
$$

In Section 1, we shall construct three types of fundamental system (nonexplicitly mixed, continuous, matching) for equation (1). In Section 2 , we shall establish the forms of three types of solutions (nonexplicitly mixed, continuous, matching) of equation (2).

\section{Construction of fundamental systems for $\left(L_{1}, L_{2}\right) f=0$}

Let us recall the following definitions from [6].

Definition 1. We call a complex valued function $f$, defined on the interval $I$, a solution (nonexplicitly mixed) of the differential equation (2) if

(i) $f / I_{1}=x \in A C^{n}\left(I_{1}\right)$,

(ii) $x$ satisfies the equation $L_{1} x=g_{1}$ for almost all $t \in I_{1}$, and

(iii) $f / I_{2}=y \in A C^{m}\left(I_{2}\right)$,

(iv) $y$ satisfies the equation $L_{2} y=g_{2}$ for almost all $t \in I_{2}$.

Definition 2. We call a complex valued function $f$ defined on the interval $I$, a continuous solution of the differential equation (2) if

(i) $f$ is a solution of equation (2) in the sense of Definition 1 , and 
(ii) the functions $f / I_{1}=x$ and $f / I_{2}=y$ satisfy continuity conditions at the interface point $t=b$, namely,

$$
x^{(j)}(b-)=y^{(j)}(b+), \quad j=0,1, \ldots, m-1 .
$$

Definition 3. We call a complex valued function $f$ defined on the interval $I$, a matching solution of the differential equation (2) if

(i) $f$ is a solution of equation (2) in the sense of Definition 1, and

(ii) the functions $f / I_{1}=x$ and $f / I_{2}=y$ satisfy the matching conditions at the interface point $t=b$, given by

$$
A \tilde{x}(b-)=B \tilde{\tilde{y}}(b+) \text {. }
$$

REMARK 1. All the above definitions carry over to equation (1) also.

Definition 4. We say that the nontrivial functions $f_{1}, \ldots, f_{p}$ defined on $I$ (with possible jump discontinuities at $t=b$ ) are linearly independent if for any set of scalars $c_{1}, \ldots, c_{p}$

$$
\sum_{i=1}^{p} c_{i} f_{i}(t)=0 \text { for all } t \in I, \quad t \neq b,
$$

and

$$
\sum_{i=1}^{p} c_{i} f_{i}(b-)=\sum_{i=1}^{b} c_{i} f_{i}(b+)=0
$$

imply $c_{1}=c_{2}=\cdots=c_{p}=0$.

Definition 5. By a fundamental system (FS) (nonexplicitly mixed, continuous, matching) for equation (1), we mean a set of linearly independent solutions (nonexplicitly mixed, continuous, matching) of equation (1) which span the solution (nonexplicitly mixed, continuous, matching) space of equation (1).

(I) Nonexplicitly mixed fundamental system for $\left(L_{1}, L_{2}\right) f=0$

Let $\alpha_{1}, \ldots, \alpha_{n}$ be a basis for $\mathbb{C}^{n}$ and $\beta_{1}, \ldots, \beta_{m}$ be a basis for $\mathbb{C}^{m}$. Let $t_{i} \in I_{i}, i=1,2$. Let $x_{i} \in A C^{n}\left(I_{1}\right)$ be the unique solution of $L_{1} x_{i}=0$, $\tilde{x}_{i}\left(t_{1}\right)=\alpha_{i}, i=1, \ldots, n$, and let $y_{i} \in A C^{m}\left(I_{2}\right)$ be the unique solution of $L_{2} y_{i}=0, \tilde{y}_{i}\left(t_{2}\right)=\beta_{i}, i=1, \ldots, m$. Clearly, $x_{1}, x_{2}, \ldots, x_{n}$ form a $F S$ for $L_{1} x=0$, and $y_{1}, \ldots, y_{m}$ form a $F S$ for $L_{2} y=0$. Define

$$
\begin{aligned}
f_{i}(t) & = \begin{cases}x_{i}(t), & t \in I_{1}, \\
0, & t \in I_{2}, t \neq b, i=1, \ldots, n,\end{cases} \\
f_{n+i}(t) & = \begin{cases}0, & t \in I_{1}, t \neq b, \\
y_{i}(t), & t \in I_{2}, i=1,2, \ldots, m .\end{cases}
\end{aligned}
$$


Then as shown in [6, Theorem 4], it can be verified that $f_{1}, \ldots, f_{n}, f_{n+1}$, $\ldots, f_{n+m}$ form a nonexplicitly mixed fundamental system for $\left(L_{1}, L_{2}\right) f=$ 0 .

Note 1. We note that the Wronskian of $x_{1}, \ldots, x_{n}$, namely,

$$
W\left(x_{1}, \ldots, w_{n}\right)(t) \neq 0
$$

for all $t \in I_{1}$, and the Wronskian of $y_{1}, \ldots, y_{m}$, namely, $W\left(y_{1}, \ldots, y_{m}\right)(t)$ $=W\left(f_{n+1}, \ldots, f_{n+m}\right)(t) \neq 0$ for all $t \in I_{2}$. Let $W_{i}\left(f_{1}, \ldots, f_{n}\right)(t)$ denote the determinant obtained from $W\left(f_{1}, \ldots, f_{n}\right)(t)$ by replacing the $i$ th column by $(0,0, \ldots, 0,1) \in \mathbb{C}^{n}, i=1, \ldots, n$, and let $W_{i}\left(f_{n+1}, \ldots, f_{n+m}\right)(t)$ denote the determinant obtained from $W\left(f_{n+1}, \ldots, f_{n+m}\right)(t)$ by replacing the $i$ th column by $(0, \ldots, 0,1) \in \mathbb{C}^{m}, i=1, \ldots, m$.

(II) Continuous fundamental system for $\left(L_{1}, L_{2}\right) f=0$

Let $\beta_{1}, \ldots, \beta_{m}$ be a basis for $\mathbb{C}^{m}$ and let $\gamma_{1}, \ldots, \gamma_{n-m}$ be a basis for $\mathbb{C}^{n-m}$. Then the vectors $\alpha_{1}, \ldots, \alpha_{n}$ defined by

$$
\alpha_{i}=\left\{\begin{array}{lr}
\operatorname{column}\left(\beta_{i}, 0\right), & 0 \in \mathbb{C}^{n-m}, i=1, \ldots, m, \\
\operatorname{column}\left(0, \gamma_{i-m}\right), & 0 \in \mathbb{C}^{m}, i=m+1, \ldots, n,
\end{array}\right.
$$

form a basis for $\mathbb{C}^{n}$. Let $x_{i} \in A C^{n}\left(I_{1}\right)$ be the unique solution of $L_{1} x_{i}=0$, $\tilde{x}_{i}(b-1)=\alpha_{i}, i=1, \ldots, n$. Let $y_{i} \in A C^{m}\left(I_{2}\right)$ be the unique solution of $L_{2} y_{i}=0, \tilde{\tilde{y}}_{i}(b+)=\beta_{i}, i=1, \ldots, m$. Clearly, $x_{1}, \ldots, x_{n}$ form a $F S$ for $L_{1} x=0$, and $y_{1}, \ldots, y_{m}$ form a $F S$ for $L_{2} y=0$. Define

$$
\begin{aligned}
f_{i}(t) & = \begin{cases}x_{i}(t), & t \in I_{1}, t \neq b, \\
y_{i}(t), & t \in I_{2}, i=1, \ldots, m,\end{cases} \\
& = \begin{cases}x_{i}(t), & t \in I_{1}, \\
0, & t \in I_{2}, t \neq b, i=m+1, \ldots, n .\end{cases}
\end{aligned}
$$

Then as shown in [6, Theorem 5], it can be verified that $f_{1}, \ldots, f_{m}, f_{m+1}$, $\ldots, f_{n}$ form a continuous fundamental system for $\left(L_{1}, L_{2}\right) f=0$.

Note 2. We note that $W\left(f_{1}, \ldots, f_{n}\right)(t) \neq 0$ for all $t \in I_{1}$, and

$$
W\left(f_{1}, \ldots, f_{m}\right)(t) \neq 0
$$

for all $t \in I_{2}$. Let $W_{i}\left(f_{1}, \ldots, f_{n}\right)(t)$ denote the determinant obtained from $W\left(f_{1}, \ldots, f_{n}\right)(t)$ by replacing the $i$ th column by $(0, \ldots, 0,1) \in \mathbb{C}^{n}, i=$ $1, \ldots, n$, and let $W_{i}\left(f_{1}, \ldots, f_{m}\right)(t)$ denote the determinant obtained from

$$
W\left(f_{1}, \ldots, f_{m}\right)(t)
$$

by replacing the $i$ th column by $(0, \ldots, 0,1) \in \mathbb{C}^{m}, i=1, \ldots, m$.

(III) Matching fundamental system for $\left(L_{1}, L_{2}\right) f=0$

Since $\operatorname{rank} A=\operatorname{rank} B=d(\leq m)$, there exist bases $\alpha_{1}, \ldots, \alpha_{n}$ for $\mathbb{C}^{n}$ and $\beta_{1}, \ldots, \beta_{m}$ for $\mathbb{C}^{m}$ such that $\alpha_{1}, \ldots, \alpha_{n-d}$ belong to the null space 
of $A$ and $\beta_{1}, \ldots, \beta_{m-d}$ belong to the null space of $B$. Also since the range of $A$ equals the range of $B$,

$$
\operatorname{span}\left\{A \alpha_{i}\right\}_{i=n-d+1}^{n}=\operatorname{span}\left\{B \beta_{i}\right\}_{i=m-d+1}^{m} .
$$

Let $x_{i} \in A C^{n}\left(I_{1}\right)$ be the unique solution of $L x_{i}=0, \tilde{x}_{i}(b-)=\alpha_{i}$, $i=1, \ldots, n$, and let $y_{i} \in A C^{m}\left(I_{2}\right)$ be the unique solution of $L_{2} y_{i}=$ $0, \tilde{\tilde{y}}_{i}(b+)=\beta_{i}, i=1, \ldots, m$. By relation (3), we get that $A \alpha_{i}=$ $\sum_{j=m-d+1}^{m} \theta_{j}^{i} B \beta_{j}, i=n-d+1, \ldots, n$, where $\theta_{j}^{i}$ are some suitable scalars. Clearly, $x_{1}, \ldots, x_{n}$ form a $F S$ for $L_{1} x=0$. Define

$$
f_{i}(t)=\left\{\begin{array}{l}
x_{i}(t), \quad t \in I_{1}, \\
0, \quad t \in I_{2}, t \neq b, i=1, \ldots, n-d, \\
x_{i}(t), \quad t \in I_{1}, t \neq b, \\
\sum_{j=m-d+1}^{m} \theta_{j}^{i} y_{j}(t), \quad t \in I_{2}, i=n-d+1, \ldots, n,
\end{array}\right.
$$

and

$$
f_{n+i}(t)=\left\{\begin{array}{lr}
0, & t \in I_{1}, t \neq b, \\
y_{i}(t), & t \in I_{2}, i=1, \ldots, m-d .
\end{array}\right.
$$

It was shown in [6, Theorem 6] that $f_{1}, \ldots, f_{n}, f_{n+1}, \ldots, f_{n+m-d}$ form a matchingly mixed fundamental system for $\left(L_{1}, L_{2}\right) f=0$.

ClaIM. $y_{1}, \ldots, y_{m-d}, \sum_{j=m-d+1}^{m} \theta_{j}^{n-d+1} y_{j}, \ldots, \sum_{j=m-d+1}^{m} \theta_{j}^{n} y_{j}$ form a fundamental system for $L_{2} y=0$.

It is enough to verify that the above functions are linearly independent, so let $c_{1}, \ldots, c_{m}$ be scalars such that

$$
\begin{gathered}
c_{1} y_{1}+\cdots+c_{m-d} y_{m-d}+c_{m-d+1} \sum_{j=m-d+1}^{m} \theta_{j}^{n-d+1} y_{j} \\
+\cdots+c_{m} \sum_{j=m-d+1}^{m} \theta_{j}^{n} y_{j}=0
\end{gathered}
$$

Upon differentiation, relation (4) implies that

$$
\begin{gathered}
c_{1} \tilde{\tilde{y}}(b+)+\cdots+c_{m-d} \tilde{\tilde{y}}_{m-d}(b+)+c_{m-d+1} \sum_{j=m-d+1}^{m} \theta_{j}^{n-d+1} \tilde{y}_{j}(b+) \\
+\cdots+c_{m} \sum_{j=m-d+1}^{m} \theta_{j}^{n} \tilde{y}_{j}(b+)=0 .
\end{gathered}
$$

Operating the matrix $B$ on both sides of equation (5), we get that

(6) $c_{m-d+1} B\left(\sum_{j=m-d+1}^{m} \theta_{j}^{n-d+1} \tilde{\tilde{y}}_{j}(b+)\right)+\cdots+c_{m} B\left(\sum_{j=m-d+1}^{m} \theta_{j}^{n} \tilde{y}_{j}(b+)\right)=0$. 
Since $f_{1}, \ldots, f_{n+m-d}$ form a matching fundamental system for $\left(L_{1}, L_{2}\right) f=$ 0 , we get from relation (6) that

$$
c_{m-d+1} A \tilde{x}_{n-d+1}(b-)+\cdots+c_{m} A \tilde{x}_{n}(b-)=0,
$$

that is,

$$
A\left(x_{m-d+1} \tilde{x}_{n-d+1}(b-)+\cdots+c_{m} \tilde{x}_{n}(b-)\right)=0,
$$

that is,

$$
A\left(c_{m-d+1} \alpha_{n-d+1}+\cdots+c_{m} \alpha_{n}\right)=0,
$$

which implies that $c_{m-d+1} \alpha_{n-d+1}+\cdots+c_{m} \alpha_{n}$ belongs to the null space of $A$ and this is true only if $c_{m-d+1}=\cdots c_{m}=0$. Now, relation (4) reduces to $c_{1} y_{1}+\cdots+c_{m-d} y_{m-d}=0$, and since $y_{1}, \ldots, y_{m-d}$ are linearly independent, we get that $c_{1}=\cdots=c_{m-d}=0$. This proves the claim.

NoTE 3. We note that $W\left(f_{1}, \ldots, f_{n}\right)(t) \neq 0$ for all $t \in I_{1}$, and

$$
W\left(f_{n-d+1}, \ldots, f_{n}, f_{n+1}, \ldots, f_{n+m-d}\right)(t) \neq 0
$$

for all $t \in I_{2}$. Let $W_{i}\left(f_{1}, \ldots, f_{n}\right)(t)$ denote the determinant obtained from $W\left(f_{1}, \ldots, f_{n}\right)(t)$ by replacing the $i$ th column by $(0, \ldots, 0,1) \in \mathbb{C}^{n}, i=$ $1,2, \ldots, n$, and let $W_{i}\left(f_{n-d+1}, \ldots, f_{n}, f_{n+1}, \ldots, f_{n+m-d}\right)(t)$ denote the determinant obtained from $W\left(f_{n-d+1}, \ldots, f_{n+m-d}\right)(t)$ by replacing the $i$ th column by $(0, \ldots, 0,1) \in \mathbb{C}^{m}, i=1, \ldots, m$.

\section{Forms of solutions of $\left(L_{1}, L_{2}\right) f=g$}

THEOREM 1 (Variation of constants method for nonexplicitly mixed solutions of $\left.\left(L_{1}, L_{2}\right) f=g\right)$. Let $f_{1}, \ldots, f_{n+m}$ be the nonexplicitly mixed fundamental system for $\left(L_{1}, L_{2}\right) f=0$ as defined in (I). Then all nonexplicitly mixed solutions $f$ of $\left(L_{1}, L_{2}\right) f=g$ are of the form $f(t)=$ $\sum_{i=1}^{n+m} c_{i}(t) f_{i}(t), t \in I$, where

$$
\begin{gathered}
c_{i}(t)=\left\{\begin{array}{l}
a_{i}+\int_{t_{1}}^{t}\left(\frac{W_{i}\left(f_{1}, \ldots, f_{n}\right)(s)}{P_{n}(s) W\left(f_{1}, \ldots, f_{n}\right)(s)} r_{1}(s) g_{1}(s) d s, \quad t \in I_{1},\right. \\
0, \quad t \in I_{2}, t \neq b, i=1, \ldots, n,
\end{array}\right. \\
c_{n+i}(t)=\left\{\begin{array}{l}
0, \quad t \in I_{1}, t \neq b, \\
b_{i}+\int_{t_{2}}^{t}\left(\frac{W_{i}\left(f_{n+1}, \ldots, f_{n+m}\right)(s)}{Q_{m}(s) W\left(f_{n+1}, \ldots, f_{n+m}\right)(s)}\right) r_{2}(s) g_{2}(s) d s, \\
t \in I_{2}, i=1, \ldots, m,
\end{array}\right.
\end{gathered}
$$

and $a_{i}(i=1, \ldots, n), b_{i}(i=1, \ldots, m)$ are scalars, and $t_{i} \in I_{i}, i=1,2$. 
Proof. By [6, Theorem 1], there exist nontrivial nonexplicitly mixed solutions of equation (2). Let us assume a nonexplicitly mixed solution $f$ of equation (2) to be of the form

$$
f(t)=\sum_{i=1}^{n+m} c_{i}(t) f_{i}(t), \quad t \in I,
$$

where the $c_{i}$ 's are to be determined by the variation of constants method.

Case (1): for $t \in I_{1}, f(t)=\sum_{i=1}^{n} c_{i}(t) f_{i}(t)=\sum_{i=1}^{n} c_{i}(t) x_{i}(t)$ satisfies the equation $L_{1} f=g_{1}$.

Therefore, by the variation of constants method we get that $c_{i}(t)=a_{i}(t)+\int_{t_{1}}^{t}\left(\frac{W_{i}\left(f_{1}, \ldots, f_{n}\right)(s)}{P_{n}(s) W\left(f_{1}, \ldots, f_{n}\right)(s)}\right) r_{1}(s) g_{1}(s) d s, \quad i=1, \ldots, n$,

where the $a_{i}$ 's are scalars and $t_{1} \in I_{1}$ (see [2, Theorem 3.6.4]). Extend the functions $c_{i}$ to the interval $I_{2}$ by defining $c_{i}(t)=0, t \in I_{2}, t \neq b$, $i=1,2, \ldots, n$.

Case (2): for $t \in I_{2}$,

$$
f(t)=\sum_{i=n+1}^{n+m} c_{i}(t) f_{i}(t)=\sum_{i=1}^{m} c_{n+i}(t) y_{i}(t)
$$

satisfies the equation $L_{2} f=g_{2}$.

Therefore by the variation of constants method, we get that

$$
\begin{array}{rl}
c_{n+i}(t)=b_{i}+\int_{t_{2}}^{t}\left(\frac{W_{i}\left(f_{n+1}, \ldots, f_{n+m}\right)(s)}{Q_{m}(s) W\left(f_{n+1}, \ldots, f_{n+m}\right)(s)}\right) r_{2}(s) g_{2}(s) d s, & \\
i & i=1,2, \ldots, m,
\end{array}
$$

where the $b_{i}$ 's are scalars and $t_{2} \in I_{2}$. Extend the functions $c_{n+i}$ to the interval $I_{1}$ by defining $c_{n+i}(t)=0, t \in I_{1}, t \neq b, i=1, \ldots, m$. Finally, recalling the definitions of $f_{1}, \ldots, f_{n}, \ldots, f_{n+m}$, we see that the proof is complete.

THEOREM 2 (Variation of constants method for continuous solutions of $\left.\left(L_{1}, L_{2}\right) f=g\right)$. Let $f_{1}, \ldots, f_{n}$ be the continuous fundamental system for $\left(L_{1}, L_{2}\right) f=0$ as defined in (II). Then all continuous solutions of $\left(L_{1}, L_{2}\right) f=$ $g$ are of the form

$$
f(t)=\sum_{i=1}^{n} c_{i}(t) f_{i}(t), \quad t \in I
$$


where

$$
c_{i}(t)=\left\{\begin{array}{c}
a_{i}+\int_{t_{1}}^{t}\left(\frac{W_{i}\left(f_{1}, \ldots, f_{n}\right)(s)}{P_{n}(s) W\left(f_{1}, \ldots, f_{n}\right)(s)}\right) r_{1}(s) g_{1}(s) d s, \quad t \in I_{1}, \\
a_{i}+\int_{t_{1}}^{b}\left(\frac{W_{i}\left(f_{1}, \ldots, f_{n}\right)(s)}{P_{n}(s) W\left(f_{1}, \ldots, f_{n}\right)(s)}\right) r_{1}(s) g_{1}(s) d s \\
+\int_{b}^{t}\left(\frac{W_{i}\left(f_{1}, \ldots, f_{m}\right)(s)}{Q_{m}(s) W\left(f_{1}, \ldots, f_{m}\right)(s)}\right) r_{2}(s) g_{2}(s) d s, \\
\quad t \in I_{2}, i=1, \ldots, m, \\
0, \quad t \in I_{2}, t \neq b, i=m+1, \ldots, n,
\end{array}\right.
$$

and $a_{i}, i=1, \ldots, n$, are scalars and $t_{1} \in I_{1}$.

Proof. By [6, Theorem 2], there exist nontrivial continuous solutions of equation (2). Let us assume a continuous solution $f$ of equation (2) to be of the form

$$
f(t)=\sum_{i=1}^{n} c_{i}(t) f_{i}(t), \quad t \in I,
$$

where the $c_{i}$ 's are to be determined by the variation of constants method.

Case (1): for $t \in I_{1}, f(t)=\sum_{i=1}^{n} c_{i}(t) f_{1}(t)=\sum_{i=1}^{n} c_{i}(t) x_{i}(t)$ satisfies the equation $L_{1} f=g$.

Therefore, by the variation of constants method we get that

$$
c_{i}(t)=a_{i}+\int_{t_{1}}^{t}\left(\frac{W_{i}\left(f_{1}, \ldots, f_{n}\right)(s)}{P_{n}(s) W\left(f_{1}, \ldots, f_{n}\right)(s)}\right) r_{1}(s) g_{1}(s) d s, \quad i=1, \ldots, n,
$$

where the $a_{i}$ 's are scalars and $t_{1} \in I_{1}$. We notice that the $c_{i}$ 's satisfy the relation

$$
\sum_{i=1}^{n} c_{i}^{(1)}(t) f_{i}^{(j)}(t)=0, \quad t \in I_{1}, j=0,1, \ldots, n-2 .
$$

Case (2): for $t \in I_{2}, f(t)=\sum_{i=1}^{m} c_{i}(t) f_{i}(t)=\sum_{i=1}^{m} c_{i}(t) y_{i}(t)$ satisfies the equation $L_{2} f=g_{2}$.

Therefore, by the variation of constants method we get that (10)

$$
c_{i}(t)=b_{i}+\int_{b}^{t}\left(\frac{W_{i}\left(f_{1}, \ldots, f_{m}\right)(s)}{Q_{m}(s) W\left(f_{1}, \ldots, f_{m}\right)(s)}\right) r_{2}(s) g_{2}(s) d s, \quad i=1, \ldots, m,
$$

where the $b_{i}$ 's are scalars. We notice that $c_{1}, \ldots, c_{m}$ satisfy the relation

$$
\sum_{i=1}^{m} c_{i}^{(1)} f_{i}^{(j)}(t)=0, \quad t \in I_{2}, j=0, \ldots, m-2 .
$$


Since $f$ is a continuous solution of equation (2), we have that $f^{(j)}(b-)=$ $f^{(j)}(b+), j=0,1, \ldots, m-1$. Therefore, from relation (7), using relations (9) and (11), we get that

$$
\sum_{i=1}^{n} c_{i}(b-) f_{i}^{(j)}(b-)=\sum_{i=1}^{m} c_{i}(b+) f_{i}^{(j)}(b+), \quad j=0,1, \ldots, m-1,
$$

which implies that

$$
\sum_{i=1}^{m} c_{i}(b-) \operatorname{column}\left(f_{i}(b-), \ldots, f_{i}^{(m-1)}(b-)\right)=\sum_{i=1}^{m} c_{i}(b+) \tilde{\tilde{f}}_{i}(b+),
$$

that is, (see (II)),

$$
\sum_{i=1}^{m} c_{i}(b-) \beta_{i}=\sum_{i=1}^{m} c_{i}(b+) \beta_{i}
$$

Now, since $\beta_{1}, \ldots, \beta_{m}$ are linearly independent, we get from relation (12) that $c_{i}(b-)=c_{i}(b+), i=1, \ldots, m$. Hence we choose the $b_{i}$ 's such that

$$
b_{i}=a_{i}+\int_{t_{1}}^{t}\left(\frac{W_{i}\left(f_{1}, \ldots, f_{n}\right)(s)}{P_{n}(s) W\left(f_{1}, \ldots, f_{n}\right)(s)}\right) r_{1}(s) g_{1}(s) d s, \quad i=1, \ldots, m .
$$

Substituting the values of $b_{i}$ 's from relation (13) into relation (10), and extending $c_{m+1}, \ldots, c_{n}$ to the interval $I_{2}$ by defining

$$
c_{i}(t)=0, \quad t \in I_{2}, t \neq b, i=m+1, \ldots, n,
$$

we see in view of relations (8), (10), (13), (14) and the definitions of $f_{1}, \ldots$, $f_{n}$ that the proof is complete.

THEOREM 3 (Variation of constants method for matching solutions of $\left.\left(L_{1}, L_{2}\right) f=g\right)$. Let $f_{1}, \ldots, f_{n+m-d}$ be the matching fundamental system for $\left(L_{1}, L_{2}\right) f=0$ as defined in (III). Then all matching solutions $f$ of $\left(L_{1}, L_{2}\right) f=g$ are of the form

$$
f(t)=\sum_{i=1}^{n+m-d} c_{i}(t) f_{i}(t), \quad t \in I
$$


where

$$
\begin{aligned}
& \left(a_{i}+\int_{t_{1}}^{t}\left(\frac{W_{i}\left(f_{1}, \ldots, f_{n}\right)(s)}{P_{n}(s) W\left(f_{1}, \ldots, f_{n}\right)(s)}\right) r_{1}(s) g_{1}(s) d s,\right. \\
& c_{i}(t)=\left\{\begin{array}{l}
0, \quad t \in I_{2}, t \neq b, i=1, \ldots, n-d, \\
a_{i}+\int_{t_{1}}^{b}\left(\frac{W_{i}\left(f_{1}, \ldots, f_{n}\right)(s)}{P_{n}(s) W\left(f_{1}, \ldots, f_{n}\right)(s)}\right) r_{1}(s) g_{1}(s) d s \\
+\int_{b}^{t}\left(\frac{W_{i}\left(f_{n-d+1}, \ldots, f_{n+m-d}\right)(s)}{Q_{m}(s) W\left(f_{n-d+1}, \ldots, f_{n+m-d}\right)(s)}\right) r_{2}(s) g_{s}(s) d s, \\
t \in I_{2}, i=n-d+1, \ldots, n,
\end{array}\right. \\
& c_{n-d+i}(t)=\left\{\begin{array}{l}
0, \quad t \in I_{1}, t \neq b, \\
b_{i}+\int_{b}^{t}\left(\frac{W_{i}\left(f_{n-d+1}, \ldots, f_{n+m-d}\right)(s)}{Q_{m}(s) W\left(f_{n-d+1}, \ldots, f_{n+m-d}\right)(s)}\right) r_{2}(s) g_{2}(s) d s, \\
t \in I_{2}, i=d+1, \ldots, m,
\end{array}\right.
\end{aligned}
$$

and $a_{i}(i=1, \ldots, n), b_{i}(i=1, \ldots, m)$ are scalars, and $t_{1} \in I_{1}$.

Proof. By [6, Theorem 3], there exist nontrivial matching solutions of equation (2). Let us assume a matching solution $f$ of equation (2) to be of the form

$$
f(t)=\sum_{i=1}^{n+m-d} c_{i}(t) f_{i}(t), \quad t \in I,
$$

where the $c_{i}$ 's are to be determined by the variation of constants method.

Case (1): for $t \in I_{1}$,

$$
f(t)=\sum_{i=1}^{n+m-d} c_{i}(t) f_{i}(t)=\sum_{i=1}^{n} c_{i}(t) f_{i}(t)=\sum_{i=1}^{n} c_{i}(t) x_{i}(t)
$$

satisfies the equation $L_{1} f=0$. Therefore, by the variation of constants method we get that

$$
c_{i}(t)=a_{i}+\int_{t_{1}}^{t}\left(\frac{W_{i}\left(f_{1}, \ldots, f_{n}\right)(s)}{P_{n}(s) W\left(f_{1}, \ldots, f_{n}\right)(s)}\right) r_{1}(s) g_{1}(s) d s, \quad i=1, \ldots, n,
$$

where the $a_{i}$ 's are scalars and $t_{1} \in I_{1}$. We notice that the $c_{i}$ 's satisfy the relation

$$
\sum_{i=1}^{n} c_{i}^{(1)}(t) f_{i}^{(j)}(t)=0, \quad t \in I_{1}, j=0,1, \ldots, n-2
$$


Case (2): for $t \in I_{2}$,

$$
\begin{aligned}
f(t) & =\sum_{i=1}^{n+m-d} c_{i}(t) f_{i}(t)=\sum_{i=n-d+1}^{n+m-d} c_{i}(t) f_{i}(t) \\
& =\sum_{i=n-d+1}^{n} c_{i}(t) \sum_{j=m-d+1}^{m} \theta_{j}^{i} y_{i}(t)+\sum_{i=1}^{m-d} c_{n+i}(t) y_{i}(t)
\end{aligned}
$$

satisfies the equation $L_{2} f=g_{2}$. Therefore, by the variation of constants method we get that

$$
\begin{array}{r}
c_{n-d+i}(t)=b_{i}+\int_{b}^{t}\left(\frac{W_{i}\left(f_{n-d+1}, \ldots, f_{n+m-d}\right)(s)}{Q_{m}(s) W\left(f_{n-d+1}, \ldots, f_{n+m-d}\right)(s)}\right) r_{2}(s) g_{2}(s) d s \\
i=1,2, \ldots, m
\end{array}
$$

where the $b_{i}$ 's are scalars. We notice that $c_{i}, i=n-d+1, \ldots, n+m-d$ satisfy the relation

$$
\sum_{i=n-d+1}^{n+m-d} c_{i}^{(1)} f_{i}^{(j)}(t)=0, \quad t \in I_{2}, j=0, \ldots, m-2
$$

Since $f$ is a matching solution of equation (2), we have that $A \tilde{f}(b-)=$ $B \tilde{\tilde{f}}(b+)$. Therefore, from relation (15), using relations (17) and (19), we get that

$$
\begin{aligned}
A f(b-) & =\sum_{i=1}^{n} c_{i}(b-) A \tilde{f}_{i}(b-)=\sum_{i=n-d+1}^{n} c_{i}(b-) A \tilde{f}_{i}(b-)=B \tilde{\tilde{f}}(b+) \\
& =\sum_{i=n-d+1}^{n+m-d} c_{i}(b+) B \tilde{\tilde{f}}_{i}(b-)=\sum_{i=n-d+1}^{n} c_{i}(b+) B \tilde{\tilde{f}}_{i}(b+),
\end{aligned}
$$

which implies that

$$
\sum_{i=n-d+1}^{n} c_{i}(b-) A \tilde{f}_{i}(b-)=\sum_{i=n-d+1}^{n} c_{i}(b+) A \tilde{f}_{i}(b+)
$$

$$
\text { (since } \left.A \tilde{f}_{i}(b-)=B \tilde{\tilde{f}}_{i}(b+)\right),
$$

that is,

$$
A\left(\sum_{i=n-d+1}^{n}\left(c_{i}(b-)-c_{i}(b+)\right) \tilde{f}_{i}(b-)\right)=0
$$

that is,

$$
A\left(\sum_{i=n-d+1}^{n}\left(c_{i}(b-)-c_{i}(b+)\right) \alpha_{i}\right)=0 \quad(\text { see }(\text { III }))
$$


Now, relation (20) implies that $\sum_{i=n-d+1}^{n}\left(c_{i}(b-)-c_{i}(b+)\right) \alpha_{i}$ belongs to the null space of $A$ and this is the true only if $c_{i}(b-)=c_{i}(b+), i=n-d+$ $1, \ldots, n$. Hence we choose $b_{i}(i=1, \ldots, d)$ such that

$$
b_{i}=a_{n-d+i}+\int_{t_{1}}^{b}\left(\frac{W_{n-d+i}\left(f_{1}, \ldots, f_{n}\right)(s)}{P_{n}(s) W\left(f_{1}, \ldots, f_{n}\right)(s)}\right) r_{1}(s) g_{1}(s) d s .
$$

Substituting these values of the $b_{i}$ 's from relation (21) into relation (18), for $i=1, \ldots, d$, and extending $c_{1}, \ldots, c_{n-d}$ to the interval $I_{2}$ by defining $c_{i}(t)=0, t \in I_{2}, t \neq b, i=1, \ldots, n-d$, and $c_{n+1}, \ldots, c_{n+m-d}$ to the interval $I_{1}$ by defining $c_{i}(t)=0, t \in I_{1}, t \neq b, i=n+1, \ldots, n+m-d$, we see in view of relations $(6),(18),(21)$ and definitions of $f_{1}, \ldots, f_{n+m-d}$ that the proof is complete.

REMARK 2. In a subsequent paper, using the results developed here, we shall construct Green's functions and generalized Green's functions for the three types of boundary value problems associated with $\left(L_{1}, L_{2}\right)$.

\section{Acknowledgements}

The authors dedicate the work to the chancellor of the institute, Bhagavan Sri Sathya Sai Baba.

\section{References}

[1] C. Allan Boyles, Acoustic waveguides, applications to oceanic sciences, (Wiley, New York, 1984).

[2] E. A. Coddington and N. Levinson, Theory of ordinary differential equations (McGrawHill, New York, 1955).

[3] P. K. Ghosh, The mathematics of waves and vibrations, (Macmillan India, Delhi, 1975).

[4] K. Noda, 'Optical fiber transmission', Studies in telecommunication, vol. 6, edited by K. Noda, (North Holland, Amsterdam, 1986).

[5] I. Stakgold, Green's functions and boundary value problems, (Wiley-Interscience, New York, 1979).

[6] M. Venkatesulu and T. Gnana Bhaskar, 'Solutions of initial value problems associated with a pair of mixed linear ordinary differential equations, in J. Math. Anal. Appl. (to appear).

[7] M. Venkatesulu and T. Gnana Bhaskar, 'Selfadjoint boundary value problems associated with a pair of mixed linear ordinary differential equations', J. Math. Anal. Appl. (to appear).

Sri Sathya Sai Insitute of Higher Learning

Prasanthinilayam-515 134, A.P.

India 